\title{
The Results of Implementation of an Acuity Tool to Decrease the Number of Resuscitation Events on an Orthopedic-Neurology Unit
}

\author{
Tanya Sobaski ${ }^{1}$, Karen Allen ${ }^{2} \&$ Samuel P. Abraham ${ }^{3}$ \\ ${ }^{1}$ Southwestern Medical Clinic, Bridgman, Michigan, USA \\ ${ }^{2}$ The College of Nursing and Health Professions, Valparaiso University, Valparaiso, IN, USA \\ ${ }^{3}$ Nursing, Bethel College School of Nursing, Mishawaka, Indiana, USA \\ Correspondence: Samuel P. Abraham, Associate Professor of Nursing, 1001 Bethel Circle, Bethel College School \\ of Nursing, Mishawaka, Indiana, USA.
}

Received: December 11, 2018

Accepted: December 15, 2018

Online Published: January 7, 2019

doi:10.20849/ijsn.v4i1.535

URL: https://doi.org/10.20849/ijsn.v4i1.535

\begin{abstract}
Background: Patients on medical-surgical units such as orthopedic-neurology are vulnerable for delayed recognition of physiological deterioration. Therefore, they are at increased risk of incurring a resuscitation event. Volume-based nurse staffing does not consider the necessity of nursing care that is individualized to the patient needs. Purpose: The purpose of this study was to implement an acuity tool to evaluate the number of resuscitation event occurrences on an Orthopedic-Neurology Unit. Method: A quasi-experimental, non-randomized, quality improvement project was implemented on a 32-bed Orthopedic/Neurology Unit over a three-month period, comparing resuscitation event occurrences with the use of the acuity tool and the group's performance without the acuity tool. Analysis: For data analysis, a Chi-square test-for-independence was used with a $2 \times 2$ contingency table, for variance of the number of patient resuscitation event occurrences with and without the use of the tool. Data were examined and combined for the three months before and after the use of the tool. When compared to the three months that the tool was employed, it yielded a $1 \%$ difference, with a $33 \%$ relative risk reduction. Results: Results indicated support for clinical significance. The use of the acuity tool demonstrated a significant decrease in the number of occurrences from 9 to 2 to 0 for each successive month of the project on the Orthopedic/Neurology Unit.
\end{abstract}

Keywords: orthopedic/neurology unit, acuity tool, staffing, CTRAT implementation, resuscitation events, patient assignments

\section{Introduction}

Missed nursing care could be traced back to inadequate nursing resources, including inadequate staffing and skill mix (Kalisch, Gosselin, \& Choi, 2012). Adequate staffing levels, quality teamwork, and good leadership improve patient outcomes. Hospital systems have been under increased pressure to improve patient outcomes and satisfaction while receiving fewer healthcare dollars for reimbursement of care, pay for performance, and cost-saving initiatives, such as bundled payments. Nursing is a service line on which it is difficult to place monetary value. There is no direct reimbursed payment for nursing services in the acute care setting. Therefore, the nursing staff is often one of the first cost centers to be cut (O'Keeffe, 2016). However, research has demonstrated that increasing nursing staff has been an effective intervention in reducing the length of stay, healthcare costs, and improving patient outcomes (Shamliyan, Kane, Mueller, Duval, \& Wilt, 2009; Twigg, Geelhoed, Bremner, \& Duffield, 2013).

\section{Background}

In a Midwestern hospital, the administrators were developing an initiative to examine how to reduce the number of resuscitation events that were occurring on medical/surgical units. The American Heart Association (AHA)-Get With the Guidelines ${ }^{\circledR}$, as part of the national Focus on Quality Program, requires hospitals to report resuscitation event occurrences (AHA, 2016). The hospital administrators decided to review the number of resuscitation events. In this hospital, nurse staffing assignments were grounded on a volume-based distribution of the patient census. Assignments were often based on convenience of the location of patient rooms. Patient assignments were made by assigning blocks of rooms to nurses usually in sequential order. Often, patients with 
high acuity were grouped together. Imbalanced workloads that do not consider the severity of the patient's illness and the required workload of the nurse can lead to less than optimal care.

\section{Purpose}

The purpose of the study was to implement Chiulli, Thompson, and Reguin-Hartman Acuity Tool (CTRAT) with measurable parameters that incorporate patient clinical severity and nurse workload indices to determine whether there was a difference in the number of resuscitation event occurrences. Hypothesis was: Implementation of the CTRAT linking patient characteristics with nurse workload will result in a difference in the number of occurrences of resuscitation event occurrences in patients on the orthopedic/neurology unit. The null hypothesis was: Implementation of the CTRAT linking patient characteristics with nurse workload will not result in a difference in the number of resuscitation event occurrences in patients on the orthopedic/neurology unit. The research question was: Will the implementation of the CTRAT result in a difference in reportable resuscitation event occurrences on an orthopedic-neurology unit of a community hospital?

\section{Methodology}

\subsection{Project Design}

Developed by nurses, the CTRAT was designed to match patient clinical severity with nurse-driven workload indices. This was a quality improvement project aimed at measuring the number of resuscitation event occurrences before and after the implementation of the acuity tool. The study was a quasi-experimental, non-randomized, convenience sample design examining data reported to the AHA. The independent variable was the implementation of the acuity tool on the Orthopedic/Neurology Unit. The dependent variables were the number of admissions to the unit and the number of resuscitation event occurrences as reported to the AHA.

\subsection{Population/Sample: Inclusion and Exclusion Criteria}

Inclusion criteria for the acuity tool were all patients admitted to the Orthopedic-Neurology Unit in an acute care facility in a mid-west rural area community teaching hospital between March 1, 2016, and November 30, 2016. The total number of admissions on the unit during the study period was 1,602. The admissions were grouped into the pre-tool application period, the application of the tool period, and the post-tool removal period.

Patients who had a do not resuscitate (DNR) or a do not intubate (DNI) status were included in the admission counts but were excluded in the number of resuscitation event occurrences. Patients transferred to other units from the Orthopedic-Neurology did not appear in the number of resuscitation events. Patients who transferred into the Orthopedic-Neurology Unit were not counted in the admission numbers but had the opportunity to be included in the number of resuscitation event occurrences.

\subsection{Setting}

The orthopedic-neurology unit was a 32-bed facility of a Community Hospital in the Midwestern United States. The unit was designed based on the Transforming Care at the Bedside Initiative that incorporated pod nursing. Pod nursing has many nurses' stations centrally located near patient rooms. The hospital used a staffing grid to determine staffing needs and placement of nurses based on patient volume census. The staffing grid called for a ratio of patients to a nurse of 5:1 on days and 6:1 on nights.

\subsection{Subjects}

The patients on the Orthopedic-Neurology Unit were adults and could be of any age over 18 . The majority of the patients were between 45 and 75 years of age. Caucasians made up the largest population of patients on the unit, with African-Americans making up the next largest racial group. There were Latino-Hispanics, Asian, and Indian ethnicities that received care on the unit. There were slightly more males than females admitted to the unit. The demographics of admissions to the Orthopedic-Neurology Unit were similar to the demographics of the surrounding community.

The staff on the Orthopedic-Neurology Unit was comprised of a mix of registered nurses and nursing assistants. There were typically six to eight registered nurses, two to three nursing assistants, and one secretary on each shift. There was one unit manager, one full-time day clinical resource nurse, and one full-time night manager. The nursing staff was comprised of "25 Associate Degree-level nurses and 29 Bachelor's in Nursing and one staff member with a Masters in nursing degree."

\subsection{Measurements/Instrumentation}

The CTRAT was used in this project to determine if it made a difference in the number of resuscitation events. Measurement for the acuity tool was an interval scale of reported resuscitation event occurrences using 
parametric testing that tracked reported resuscitation event occurrences in the three-month periods before, during, and after implementation of the tool.

Instrumentation was a revised (see Table 1) paper-based acuity tool developed by Chiulli et al. (2014) and used on a post-surgical unit at Duke Raleigh Hospital that measured "10 categories-six related to patient clinical severity and four related to nurse workload" (p. 10). The Clinical Severity Indicators categories were "Assessment, Respiratory, Cardiac, Medications and Therapeutic Protocols, Drainage Devices, Pain Management." The Nurse Workload Indicators were "Admit/DC/Transfer, Education and/or Psychological, Wound/Ostomy/Continence, ADLs and Isolation." The scoring was a ranking from 2-a "Stable Patient Typical Workload," 3-a "Complex Patient Increased Workload," to 4-being a "High-Risk Highest Workload" system (Chiulli et al., 2014, p. 10, Figure 2). The simplicity of the tool was that it required no mathematical computation. Once a parameter was met in a higher designated column, the acuity score jumped to the highest column checked. Modifications made to the CTRAT included the addition of the neurological status of the patient. The neurological status addition also included any clinical institute withdrawal assessment (CIWA) scores or delirium protocols that were instituted.

The premise of the acuity tool was to balance the workload with patient needs and nurse competencies through "appropriate staffing skill mix and staffing ratios" (Chiulli, 2014, p. 12). Empowerment of the nurses to care for patients within their competency levels through "safer nursing workload" (Chiulli et al., 2014, p. 12) led to a decreased number of resuscitation event occurrences. It was also felt that the nursing staff would be more satisfied with the quality of the care they could provide to their assigned patients.

Table 1. Revised orthopedic-neurologic unit acuity tool

\begin{tabular}{|c|c|c|c|}
\hline $\begin{array}{l}\mathrm{O} / \mathrm{N} \\
\text { Tool }\end{array}$ & $\begin{array}{l}2 \vee \text { Stable Patient } \\
\text { Typical Workload }\end{array}$ & 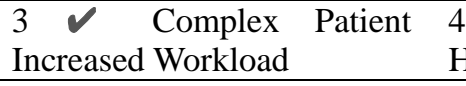 & $\begin{array}{l}4 \vee \text { High Risk } \\
\text { Highest Workload }\end{array}$ \\
\hline \multicolumn{4}{|c|}{ Clinical Severity Indicators } \\
\hline & Q4h VS/Neuro $\checkmark$, A & Q2h VS/Neuro $\checkmark$ & Q1h or $1: 1$ care, deteriorating LOC, \\
\hline Assessment & $\& O$, CIWA score $</=7$ & Confused, CIWA score >7 & Delirium (post-op/DTs) \\
\hline Respiratory & Stable & Treated $\quad \mathrm{NC}>2 \mathrm{~L}, \quad$ nasal $\mathrm{C}$ & Compromised/At Risk \\
\hline Cardiac & $\begin{array}{l}\text { Room air or } \mathrm{NC}</=2 \mathrm{~L} \mathrm{O}_{2} \\
\mathrm{BP}, \mathrm{HR} \text {, } \\
+/-15 \% \text { of baseline, } \\
\text { telemetry }\end{array}$ & $\begin{array}{ll}\text { CPAP, Continuous Pox } & \mathrm{N} \\
\text { Changes in BP/HR/Rhythm } & \mathrm{U} \\
\text { Post-op monitoring/Epidural } & \mathrm{N} \\
\text { AICD/Pacemaker } & \end{array}$ & $\begin{array}{l}\text { Masks, BiPAP, Full face CPAP } \\
\text { Unstable rhythm, } \\
\text { New Afib, freq ectopy }\end{array}$ \\
\hline $\begin{array}{l}\text { Medications/ } \\
\text { Therapeutic }\end{array}$ & $\begin{array}{l}\text { PO/IVPB, TPN, } \\
\text { BSM ac/hs/q 4-6h }\end{array}$ & $\begin{array}{l}\text { Blood administration, } \\
\text { Q 2h pain control, }\end{array}$ & $\begin{array}{l}>2 \text { transfusions, } \\
\text { fluid boluses for BP/UOP, Amiodarone, }\end{array}$ \\
\hline Protocols & $\mathrm{Mg} / \mathrm{K}^{+}$protocols & Heparin IV, CBI & $\begin{array}{l}\text { DOPAmine/DOBUtamine, } \\
\text { drip }\end{array}$ \\
\hline $\begin{array}{l}\text { Drainage } \\
\text { Devices }\end{array}$ & $\begin{array}{l}\text { JP/Hemovac, } \quad \text { NGT, } \\
\text { Thoradrain/CT }\end{array}$ & $\begin{array}{l}\text { Q2h measure of CT, } \\
\text { NG/J/G-Peg tube w/feeding }\end{array}$ & $\begin{array}{l}\text { Q1h measurement of CT, } 2 \text { CT, } \\
\text { or }>100 \mathrm{~mL} />2 \mathrm{~h} \text { CT output }\end{array}$ \\
\hline $\begin{array}{l}\text { Pain } \\
\text { Management }\end{array}$ & PO, Q4h IV, PCA & Nerve block/Epidural, & Uncontrolled pain, Multiple devices \\
\hline Nurse & & Workload & Indicators \\
\hline $\begin{array}{l}\text { Admit/DC/Tra } \\
\text { nsfer }\end{array}$ & $\begin{array}{l}\text { Post-op, Routine DC, } \\
\text { In-pt staying }\end{array}$ & $\begin{array}{l}\text { Post-op }\left(1^{\text {st }} 24 \mathrm{~h}\right), \text { Complex } \\
\text { DC, Admit/Transfer in-pt. }\end{array}$ & $\begin{array}{l}\text { xost-op (Complicated) } \\
\text { Transfer to a higher level of care, } \\
\text { Rapid Response }\end{array}$ \\
\hline $\begin{array}{l}\text { Education } \\
\text { and/or } \\
\text { Psychosocial }\end{array}$ & Calm, Cooperative & $\begin{array}{l}\text { Anxious, Service Recovery, } \\
\text { EBP Education Protocol, } \\
\text { Interpreter/Translator }\end{array}$ & $\begin{array}{l}\text { y, Highly Agitated 1:1, Extensive } \\
1, \quad \text { patient/family service recovery }\end{array}$ \\
\hline Wound/Ostom & QD/BID/Dressing by RN, & TID Dressings by RN, High & Dressing $>30 \mathrm{~min}>\mathrm{TID}$ \\
\hline $\begin{array}{l}\text { y/ } \\
\text { Continence }\end{array}$ & $\begin{array}{l}\text { Wound Vac, Ostomy, } 1 \\
\text { person assist to BR, } \\
\text { Bedpan }\end{array}$ & $\begin{array}{l}\text { Output Ostomy, Enemas or } \\
\text { Bowel Prep }\end{array}$ & $\begin{array}{l}\text { Multiple Wound Vacs, } \\
\text { Q1h toileting/Incontinence }\end{array}$ \\
\hline $\begin{array}{l}\text { ADLs and } \\
\text { Isolation }\end{array}$ & $\begin{array}{l}\text { Independent ADLs, } 1 \\
\text { person assist w/ADLs, } \\
\text { Standard Precautions } \\
\text { All 2s make a "2" }\end{array}$ & $\begin{array}{l}\text { Turn Q2h, } 2 \text { Person assist } \\
\text { OOB, } \\
\text { Isolation - all types } \\
\text { Any } 3 \text { makes a patient a “3" }\end{array}$ & $\begin{array}{l}\text { st Para/Quadraplegic } \\
\text { Total Care } \\
\text { Any } 4 \text { makes a patient a "4" }\end{array}$ \\
\hline
\end{tabular}

Adapted from Chiulli et al., 2014. Used with permission 


\section{Data Collection}

The CTRAT project was introduced and discussed with staff members a week before the training began. After training, an informal survey consisting of nine short-answer questions, along with an email was sent to the entire staff on the orthopedic-neurology unit. The instructions included anonymous feedback on the CTRAT, the perceived benefits and detriments to the tool, and its use on the unit were requested. The survey was sent to 78 people on the Orthopedic-Neurology Unit email list; this included all staff, managers, ancillary staff, and administrators. The email indicated that additional surveys were available in the break-room for ease of access. The instructions included that there was a 9 x 12 envelope placed in the break-room in the researcher's mailbox where the surveys could be returned. In the email that contained the survey, the staff was notified that the project would end on August 31 at 2300 and that their feedback would be appreciated before that deadline. The staff was encouraged to complete the survey anonymously.

Of the 78 surveys sent out approximately 50 went to the nursing staff. The staff had three weeks to submit their feedback. The survey questions asked the individuals completing the form to identify what their role was on the Orthopedic/Neurology Unit. Survey responses included four individuals who were directly affected by the implementation of the CTRAT.

Four RNs and one unit secretary responded. The survey also asked the individuals if they had used the acuity tool each shift they worked, and if not, why. Two RNs worked the evening/night shift, one RN worked the day shift, and one RN did not indicate what shift was worked. The unit secretary worked a variety of shifts. The RNs reported that they did use the acuity tool, but not always. The survey asked what barriers there were to using the Acuity Tool. Every respondent mentioned staffing issues and time constraints. One respondent also stated that there was a conflict between how the hospital staffs and acuity ratings. One respondent felt "no one should be a '2."' The next question asked what benefits the staff have seen or may see in the future for using the Acuity Tool. They responded the need for benefits to include "caps on the acuity scores and fairer workloads." One respondent replied that he/she saw no benefit that "the unit clerk asks for the patient information anyway before making the assignment." When asked what improvements could be made to the Acuity Tool, the responses were: to make the Acuity Tool "more specific and individualized to the unit;" "there needed to be a greater spread between the numbers (i.e., 2-6);" "there needs to be a cap on acuity." The next question was if there should be an Acuity Score cap. All respondents replied "yes." The following question asked what level of acuity they thought was enough without jeopardizing patient safety based on the current 2-4 score per patient. The responses were: "12, 20, 11-12, 16." The participants were thanked for completing the survey.

It was found through spot-checking and interviewing that the staff was compliant in the use of the CTRAT consistently through August. On August 31, the project ended. The staff removed the poster, the acuity tool worksheets, and the Unit Acuity Score worksheet and returned to the traditional method of making assignments and writing patient totals on the whiteboard.

There is no standardized method for escalating care. The hospital does have Vocera ${ }^{\circledR}$, a call system, in place and does track the number of times this is activated. When a "rapid response" or call for a multidisciplinary evaluation team is needed, this is recorded in the call system. However, nurses often collaborate with other nurses, medical residents, and physicians, and escalation of care is done without a broadcast call for assistance.

The facility reported data to the AHA was completed by examining medical records from rapid responses, cardiopulmonary arrest reports that were completed when the crash cart was opened, and reports about patient deterioration. Many people are involved in the process for data mining and reporting to the AHA. The process at the time of this study involved reading the electronic health record for each case.

\section{Analysis and Results}

Data analysis using Pearson Chi-square test-for-independence with a $2 \times 2$ contingency table for variance between two independent samples was conducted using IBM Statistical Analysis Data Software (SPSS) Version 22. Pearson Chi-square statistic is a test that is used to determine if there is a variance in distribution between the sample and population (UCDavis.edu, n.d.). The test-for-independence, also known as Pearson Chi-square, is useful to analyze "whether two categorical or nominal variables are related or associated with each other" or by chance (Wielkiewicz, 2000, para. 1). The $2 \times 2$ contingency table is useful in comparing the populations that received the intervention and those that did not (see Table 2).

"A Chi-square test of independence was calculated comparing the results" of patients admitted to the orthopedic/neurology unit and the number of resuscitation event occurrences with and without the CTRAT intervention on workloads and staffing (Cronk, 2008, p. 90). Data analysis showed no statistically significant 
relationship $\left(\chi^{2}(1)=1.250, p>.05\right)$. Examination of the data using purely statistical tests showed no statistical significance existed. However, there was evidence of clinical significance, which is significant when studying clinical populations.

The data examined for the statistical tests was nominal. When the data are at a nominal level, Chi-square is an effective means of discerning observed occurrences from expected occurrences between independent groups (Tappen, 2011). Data are grouped to reflect the number of occurrences between the groups. The variance in distribution between the "outcome variable among the comparison groups" supports the hypothesis (Sullivan, 2012, p. 156).

Table 2. Intervention-occurrence cross-tabulation

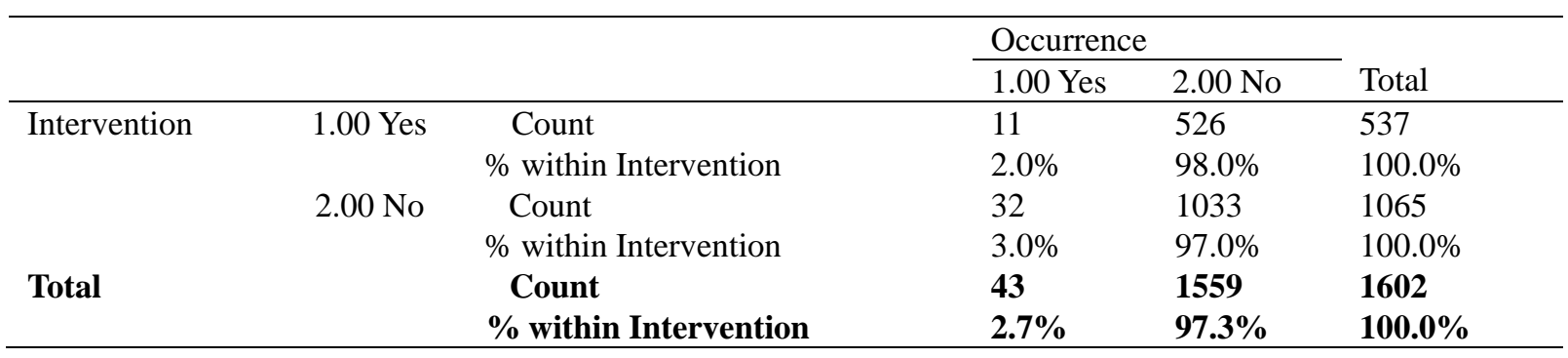

Two assumptions needed to be met for the Chi-square test to be used. The first assumption was that each cell of the table contains at least five occurrences. The second assumption was that the population and intervention have to be independent of one another (Krishnan, 2011; Tappen, 2011). Once these assumptions are met, the Chi-square test can be used effectively. The test for independence assumes that the population that received care while the CTRAT was implemented is independent of the population that received care without the acuity tool.

Using the Chi-square statistical test, the decision rule, to reject or fail to reject the null hypothesis, "depends on the level of significance and the degrees of freedom, as defined as $d f=\mathrm{k}-1$, where $k$ is the number of response categories" (Sullivan, 2012, p. 136). In the data analyzed for this study, the level of significance was 0.05 . This level of significance meant that the confidence interval of $95 \%$ had a margin of error no greater than $5 \%$. This "allows a $5 \%$ probability of incorrectly rejecting the null hypothesis in favor of the alternative when the null is true" (Sullivan, 2012, p. 125). The degrees of freedom is the number of rows and columns minus 1 from each, i.e., " $d f=(\mathrm{c}-1)(\mathrm{r}-1)$ " (Sullivan, 2012, p. 158).

Degrees of freedom allow the contingency of the data when estimating the populations. The hypothesis was whether the CTRAT would make a difference in the number of occurrences in FTR on the Orthopedic/Neurology Unit. The data analysis compared the number of admissions and the number of FTR codes with the intervention of the Acuity Tool or not. Each variable was represented in a column and row, making it a $2 \times 2$ contingency table with degrees of freedom equaling one.

Analysis of the data was completed using a Chi-square test-for-independence $2 \times 2$ contingency table. The $2 \times 2$ contingency table indicates whether the association between the population that received the intervention CTRAT and the population who did not receive the intervention is greater than it would be if by chance (Krishnan, 2011; Tappen, 2011).

The results of the analysis were Chi-square $1.250>0.05$, indicating that the use of the CTRAT was not statistically significant in reducing the number of resuscitation event occurrences on the Orthopedic/Neurology Unit. Data grouping was performed by pre- and post-implementation and the study period as with and without the CTRAT. Data collection spanned a nine-month period that was grouped in three-month increments to allow for ample sample size.

The first period of data collection was examining during the months of March, April, and May 2016, when the CTRAT was not used. During these months, acuity scoring was not done. Patient characteristics and nurse workload indicators for staffing assignments were not used. The second period of data collection occurred in June, July, and August 2016. The CTRAT was implemented and utilized during these months (see Table 3). Although there was inconsistent use of the CTRAT at the start of the project, compliance improved with time, with consistent use by the last month of the project implementation. The CTRAT was used to link the patient clinical severity with nurse workload indicators. The third period of data collection was the months of September, October, and November 2016. The CTRAT was not used during this time. The staff had stopped using the tool at 
2300 on August 31, 2016.

Table 3. Example of unit assignments worksheet

\begin{tabular}{|c|c|c|c|c|c|}
\hline \multicolumn{2}{|c|}{ Paige CN III } & \multicolumn{2}{|c|}{ Gigi CN IV } & \multicolumn{2}{|c|}{ Ashley CN II } \\
\hline Room \# & Acuity & Room \# & Acuity & Room \# & Acuity \\
\hline 5200 & 3 & 5233 & 4 & 5201 & 2 \\
\hline 5203 & 4 & 5234 & 4 & 5204 & 2 \\
\hline 5210 & 3 & 5222 & 2 & 5206 & 2 \\
\hline \multirow{2}{*}{5212} & 2 & 5221 & 3 & 5224 & 2 \\
\hline & & & & 5226 & 3 \\
\hline \multicolumn{2}{|c|}{ Helen CN IV } & \multicolumn{2}{|c|}{ Ellen CN III } & \multicolumn{2}{|c|}{ Betsy CN IV } \\
\hline Room \# & Acuity & Room \# & Acuity & Room \# & Acuity \\
\hline 5209 & 4 & 5235 & 2 & 5224 & 3 \\
\hline 5207 & 4 & 5238 & 3 & 5228 & 3 \\
\hline 5236 & 3 & 5240 & 3 & 5229 & 2 \\
\hline 5237 & 3 & 5215 & 3 & 5230 & 3 \\
\hline & & 5212 & 3 & 5232 & 3 \\
\hline
\end{tabular}

Note. Example of unit assignment sheet that is completed by the charge nurse. Patient assignments are based on acuity to balance the workload (Chiulli, 2014, p. 12). In this example, nurses are listed by their first names and level of competency or experience indicated by Roman numerals, with a II being less experienced than a IV.

For analysis, the months that the tool was not used-March, April, May, September, October, and November-were examined individually, then combined and compared with the months the project was implemented. The periods of time the tool was not in use in the population was $n=1065$. The number of reported resuscitation event occurrences was 32 . The population, when the tool was being utilized, was $n=537$. The number of reported resuscitation event occurrences was 11.

\section{Discussion}

While the data analysis indicated there was no statistical significance, there was clinical significance that emerged. The term "no statistical significance" should not dismiss the importance of data analysis that is less than the standard Cronbach's alpha $(\alpha=0.05$ or 0.01$)$ for scientific research. The clinical significance can be determined using the absolute risk reduction (ARR) and the relative risk reduction (RRR) (Polit \& Beck, 2017; Tappen, 2011).

The clinical significance of the data using the magnitude of the effect analyzed the degree of benefit versus harm. Absolute risk reduction is the percentage in reducing harm. The absolute risk (AR) of the exposed group is determined by dividing the number of resuscitation occurrence events in the group with the CTRAT (11) by the total number of patients in the exposed group (537). The result of the absolute risk exposed $\left(\mathrm{AR}_{\mathrm{E}}\right)$ was 0.02 . The $\mathrm{AR}$ of the non-exposed group $\left(\mathrm{AR}_{\mathrm{NE}}\right)$ was found by dividing the number of resuscitation occurrence events in the non-exposed group (32) by the total number of patients in the non-exposed group (1065). To find the ARR, the $\mathrm{AR}_{\mathrm{NE}}$ was subtracted from the $\mathrm{AR}_{\mathrm{E}}(0.03-0.02)$. The $\mathrm{ARR}$ for this project was $1 \%$.

The RRR is the difference in the rate of harmful outcomes. The RRR was found by dividing the ARR (0.01) by the $\operatorname{AR}_{\mathrm{NE}}(0.03)$ for a result of 0.33 . With the application of the CTRAT, the result was a $33 \%$ reduction in the rate of resuscitation event occurrences. Figure 1 demonstrates the clinical significance of the reduction of resuscitation event occurrences. 


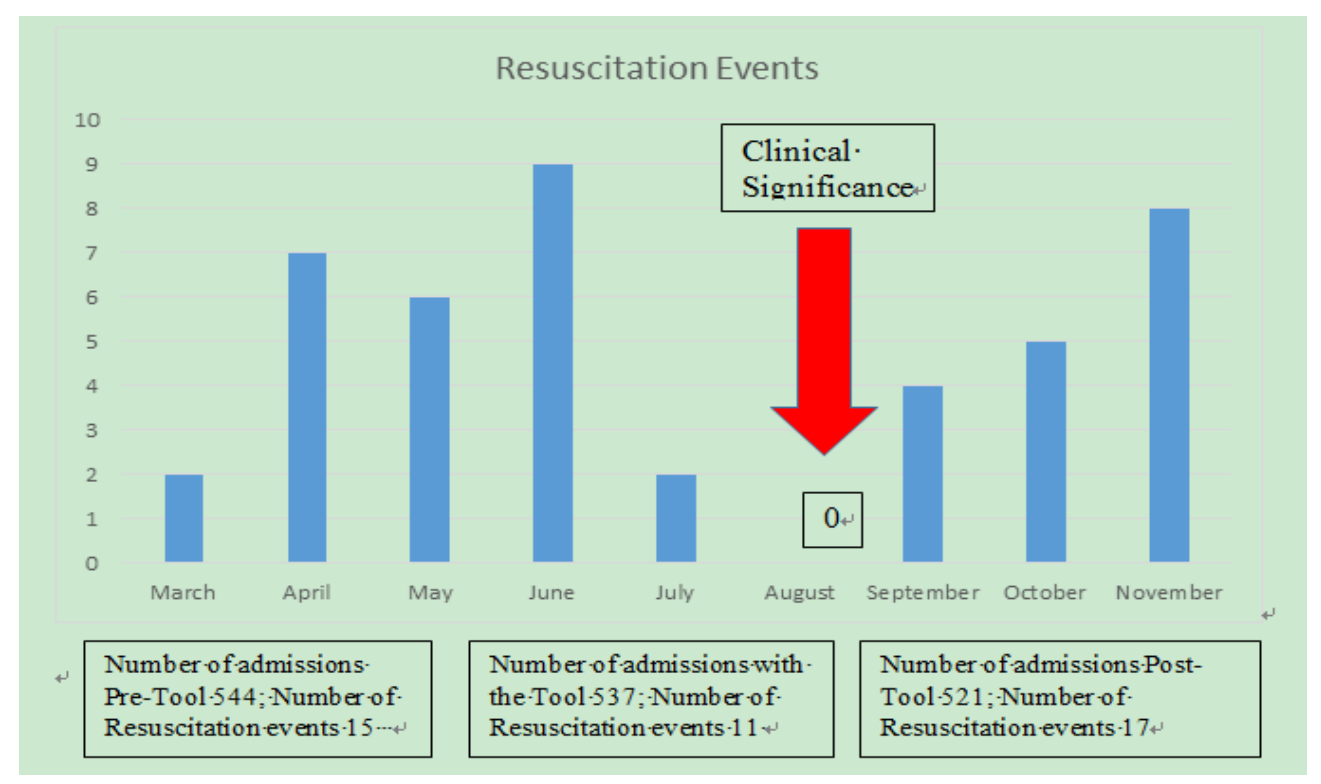

Figure 1. Resuscitation events

The months of March, April, and May were before the CTRAT was applied. The months of June, July, and August were when the tool was being used. As previously mentioned, there was consistent monitoring, education, and remediation that was occurring. In June, the month the tool was first applied, there were nine occurrences of resuscitation events. July had improved adherence and applicability and a decrease to only two resuscitation events occurrences, which was a 78\% reduction. In August, the tool was used consistently for the entire month, and no resuscitation events occurred. In the period of September, October, and November, when the tool was removed, and staffing returned to the previous methods, there was an increase in the number of events that occurred and continued on an upward trajectory over each month.

\section{Limitations}

There was a marked improvement in the reduction of resuscitation occurrence events, but the period studied was only three months. Prolonging the time the CTRAT was utilized over six months to one year would provide a complete representation of the effectiveness of the tool. Another limitation was the number of resuscitation events.

The amount of time required to complete the CTRAT took longer than reported. The acuity tool was described to take "about ten seconds per patient per shift to complete" (Chiulli et al., 2014, p. 12). The time for completion was not the case during the project. It took on average approximately 20-30 seconds per patient to complete, multiplied by five to six patients, twice during a shift, resulting in a loss of two to three minutes per shift. The bigger problem was managing to complete the acuity scoring before the staffing was set for the next shift.

The Orthopedic-Neurology Unit is a busy unit with a high patient turnover. In a 24 hour period, there are approximately one to three admissions on average and just as many discharges. Many of the surgeries start coming to the unit around noontime. The acuity scoring was to be completed by 1330 and 1730, which were often busy times on the Orthopedic/Neurology Unit. This resulted in the need for the acuity scores to be gathered by the secretary and occasionally, the charge nurses. The patient should take priority over paperwork and nurses were critical of a process that required them to complete acuity scores, which may have led to inconsistency in using objective data to score the patients' acuity.

\section{Implications}

The implications would warrant continued studies to help build the knowledge base of translational research using the application of the CTRAT. Additional ways this tool could be used are in consideration of nurse workload indicators in the development of unit specific nursing competencies. One of the challenges of this study was that the facility staffing office did not take acuity into account when determining staffing for the units. Policy development for the facility that addressed staffing needs and alignment of the nurse competencies with the patient needs which would result in positive effects. The tool could also be adapted to help with patient placement in the acute care setting.

\section{Conclusion}

The CTRAT project improved patient outcomes through the reduction in the number of resuscitation event 
occurrences. When nurses are overwhelmed by the workload in their assigned patient care groups, their ability to be aware of impending change is challenged and significantly reduced. The need to find data-driven, objective ways of determining patient clinical severity and nurse workload indicators is necessary. Acuity tools need to be simple, easy to use and have appropriate inter-rater reliability. The complex process of development of staffing assignments to ensure decreased resuscitation events, improved patient outcomes and staff satisfaction, and the equitable distribution of workload for patient care make a case for the need to use patient acuity in making assignments. The CTRAT has demonstrated that there are ways to equalize nursing assignments to balance patient clinical severity and nurse workload indicators to improve care.

\section{References}

American Heart Association (AHA). (2016). Resuscitation fact sheet. Get With The Guidelines. Retrieved from http://www.heart.org/idc/groups/heart-public/@private/@wcm/@hcm/@gwtg/documents/downloadable/uc m_434082.pdf

Chiulli, K., Thompson, J., \& Reguin-Hartman, K.L. (2014). Development and implementation of a patient acuity tool for a medical-surgical unit. Academy of Medical-Surgical Nurses, 23(2), 9-12.

Cronk, B.C. (2008). How to use SPSS: A step-by-step guide to analysis and interpretation (5th ed.). Glendale, CA: Pyrczak.

Kalisch, B.J., Gosselin, K., \& Choi, S.H. (2012). A comparison of patient care units with high versus low levels of missed nursing care. Health Care Management Review, 37(4), 320-328. https://doi.org/10.1097/HMR.0b013e318249727e

Krishnan, S. (2011). 2 key assumptions to be aware of before applying the chi-squared test. Retrieved from http://www.simafore.com/blog/bid/56480/2-key-assumptions-to-be-aware-of-before-applying-the-chi-squar e-test

O’Keeffe, M. (2016, September). Practical steps for applying acuity-based staffing. American Nurse Today, 11(9). Retrieved from https:/www.americannursetoday.com/practical-steps-applying-acuity-based-staffing/

Polit, D.F., \& Beck, C.T. (2017). Nursing Research: Generating and Assessing Evidence for Nursing Practice (10th ed.). Philadelphia, PA: Wolters Kluwer.

Shamliyan, T.A., Kane, R.L., Mueller, C., Duval, S., \& Wilt, T.J. (2009). Cost savings associated with increased RN staffing in acute care hospitals: Simulation exercise. Nursing Economics, 27(5). Retrieved from http://www.austincc.edu/nursmods/rrc/rrc_lev4/rnsg_2221/documents/Cost_Savings_Associated_with_Incr eased_RN_Staffing_module_4.pdf

Sullivan, L.M. (2012). Essentials of Biostatistics in Public Health (2nd ed.). Burlington, MA: Jones \& Bartlett Learning.

Tappen, R.M. (2011). Advanced Nursing Research. Sudbury, MA: Jones \& Bartlett Learning.

The University of California at Davis. (n.d.). Sampling distributions. Retrieved from http://pba .ucdavis.edu/files/45001.pdf

Twigg, D.E., Geelhoed, E.A., Bremner, A.P., \& Duffield, C.M. (2013). The economic benefits of increased levels of nursing care in the hospital setting. Journal of Advanced Nursing, 69(10), 2253-2261. https://doi.org/10.1111/jan.12109

Wielkiewicz, R.M. (2000). Chi-square with SPSS. In R. Runyon, K. Coleman, \& D. Pittenger (Eds.), Fundamentals of Behavioral Statistics (4th ed.). Retrieved from http://www.mhhe .com/socscience/psychology/runyon/spss/chisquare.html

\section{Copyrights}

Copyright for this article is retained by the author(s), with first publication rights granted to the journal.

This is an open-access article distributed under the terms and conditions of the Creative Commons Attribution license (http://creativecommons.org/licenses/by/4.0/). 